\title{
Interacciones pedagógicas en docentes categorizados como básicos y competentes en la evaluación docente: una mirada desde la experiencia de aprendizaje mediado (EAM).
}

\author{
Cristián Lobos Rebolledo ${ }^{1}$ \\ Ignacio Figueroa Céspedes ${ }^{2}$ \\ Jorge Soto Cárcamo ${ }^{3}$
}

\section{RESUMEN}

El presente artículo busca analizar las interacciones pedagógicas de docentes categorizados como competentes y básicos empleando una metodología mixta que triangula los datos arrojados por la evaluación docente y la observación de clases mediante pautas de observación de criterios de mediación. Se caracterizan las interacciones desde la experiencia de aprendizaje mediado, relacionando dichos resultados con las categorías obtenidas en la evaluación docente, arrojando que los docentes competentes estudiados interactúan con mejor calidad que aquellos docentes evaluados como básicos, pero ninguno de los dos grupos genera condiciones de modificabilidad cognitiva en el aula, por lo que el resultado general obtenido en la evaluación docente no presupone un tipo de interacción pedagógica cognitivamente desafiante y transformadora para los casos estudiados.

Palabras clave: Evaluación Docente, Interacciones Pedagógicas, Criterios de Mediación, Experiencia de Aprendizaje Mediado.

Pedagogical interactions of teachers categorized as basic and competent in teacher evaluation: a view from the mediated learning experience.

\section{ABSTRACT}

This article seeks to analyze the interactions of teachers teaching categorized as competent and basic using a mixed methodology that triangulate the data produced by the Educational Evaluation and Observation of classes by watching Criteria of Mediation. Interactions are characterized from the theory of Mediated Learning Experience, relating these results with the categories obtained in teacher evaluation. This analysis shows that the studied competent teachers interact with better quality than those evaluated teachers as basic but neither of the two groups creates conditions

I Magíster en Desarrollo Cognitivo, Coordinador Comunal PIE, Departamento de Educación de Los Vilos, Chileno, E-mail: coordinador.comunal.pie@gmail.com

2 Magíster en Desarrollo Cognitivo, Académico Centro de Desarrollo Cognitivo, Facultad de Educación, Universidad Diego Portales, Chileno, E-mail: ignacio.figueroa@mail.udp.cl.

3 Magíster en Investigación Sociológica Aplicada, Académico Centro de Desarrollo Cognitivo, Facultad de Educación, Universidad Diego Portales, Chileno, E-mail: jorge.sotoc@mail.udp.cl 
modifiability in the classroom, so that the overall result obtained in Teacher Evaluation does not imply a type of Pedagogical Interaction cognitively challenging and transformative.

Keywords: Teacher quality assesment, Pedagogical Interactions, Criteria Mediation, Mediated Learning Experience.

\section{Introducción}

La interacción pedagógica ha devenido en una temática de interés por distintos autores contemporáneos (Villalta y Martinic, 2009; Treviño, Toledo y Gempp, 2013; Villalta, Assael y Martinic, 20I3), relevándose su importancia en el proceso educativo. La sala de clase es el lugar donde se definen los procesos pedagógicos más relevantes en el contexto escolar y es el espacio en donde comparten y construyen conocimiento en conjunto estudiantes y profesores, expresando distintas modalidades de interacción, dando lugar a procesos de enseñanza y aprendizaje en contextos de diversidad. De este modo, lo que ocurre en el aula opera como un factor explicativo central de los aprendizajes (Slavin, 1996; LLECE, 2008; Murillo y Román, 2009, en Villalta y Martinic, 2009).

La "Interacción Pedagógica" es una de las ocho dimensiones que conforman el portafolio de la evaluación docente. Esta dimensión es entendida como la capacidad que deben tener los docentes para brindar explicaciones de calidad cuando abordan los contenidos y habilidades durante sus clases, de promover un diálogo directo con sus estudiantes de manera transversal, de realizar un monitoreo constante durante el desarrollo de las actividades y de apuntar al desarrollo del pensamiento de todos sus estudiantes, pero esta dimensión es la que refleja el menor desempeño de forma sostenida por parte de los docentes que se evalúan año a año (Manzi, González, \& Sun, 20II). El resultado en esta dimensión refleja uno de los problemas que poseen los sistemas de evaluación por ponderaciones, que muchas veces ocultan información importante en sus resultados generales (Darling-Hammond, 2012).

Uno de los objetivos de la evaluación docente declarados por la OCDE (20I3), implica consolidar las políticas orientadas al 
fortalecimiento de la función docente para mejorar la calidad y equidad de la educación. Esta instancia, ha contribuido a identificar fortalezas y debilidades de los profesores evaluados, y ha aportado un impulso a la investigación educacional en la línea de la efectividad escolar, con estudios que complementan y enriquecen el acervo en la materia. Ahora bien, desde una perspectiva crítica, de acuerdo a Monzón (2016), en la actualidad prevalece un sistema de evaluación docente que privilegia la búsqueda de semejanzas en el docente en su práctica pedagógica, denominándolo univocista. Este modelo, de raíz positivista y mecanicista, resulta insuficiente para otorgar información valiosa sobre el quehacer docente, por lo que modelos de tipo analógico podrían permitir relacionar datos cuantitativos y experiencias subjetivas, poniendo énfasis en la cualidad del contexto.

Una investigación desarrollada por un equipo liderado por Manzi (20II), a partir de los datos reunidos por los portafolios de 55.536 docentes evaluados desde el año 2006 a 2009 de todos los niveles, modalidades y subsectores educativos, arrojó que el mejor desempeño de los docentes está en el "Ambiente Instruccional” durante la clase filmada, pero por otro lado se observa un débil desempeño en la calidad de las "Interacciones Pedagógicas" entre docente y estudiante. La mayoría de las intervenciones tienden a estimular la respuesta mecánica de un procedimiento, apelando a la mera repetición de lo dicho por el mismo, o bien indican o inducen la respuesta correcta en vez de guiar un tipo de razonamiento lógico hacia la solución, cognitivamente desafiante (Manzi, J. et al., 20I I). Por otro lado, en un estudio realizado por Martinic y Vergara (2007) se da cuenta de que la interacción de los docentes con sus estudiantes está centrada en la transmisión de contenidos e instrucción de competencias con un único objetivo de obtener resultados cuantificables que reflejen el desempeño de los estudiantes, con foco en una enseñanza reproductiva que no genera desafío cognitivo. Por otra parte, Godoy (2015), investigando docentes evaluados como destacados en Historia y Geografía, determina que los participantes mayoritariamente realizan preguntas que favorecen el desarrollo de baja exigencia cognitiva, al recuperar aprendizajes ya adquiridos, vía preguntas de nivel explícito. 
En este marco, debiera uno preguntarse, ¿de qué forma están interactuando nuestros docentes? ¿Están propiciando el desarrollo del pensamiento en sus clases? El presente artículo busca conocer y caracterizar las interacciones pedagógicas, desde el enfoque de la experiencia de aprendizaje mediado (EAM), buscando vinculaciones cualitativas con los procesos de etiquetamiento y categorización docente que establece la evaluación profesional docente y estableciendo líneas de acción desde una línea analógica y de perspectiva cualitativa, pensando en el proceso de evaluación docente.

\section{Marco referencial}

\subsection{Evaluación docente e interacciones pedagógicas}

La construcción del conocimiento y el aprendizaje no se da de forma vertical, más bien supone el diálogo y la construcción conjunta entre ambos agentes que interactúan en la situación de aprendizaje (Bonal, 1998). La interacción docente-estudiante es una realidad particular con actos, saberes, hablas y expresiones concretas de aprender del estudiante y de enseñar por parte del docente (Villalta \& Martinic, 2009). Esta interacción posee pautas, sentidos, tiempos y espacios que han sido aprendidos y compartidos como "Cultura Escolar", la cual actúa como regla que ordena las posiciones e intercambios de información (Burger, 2004).

En la evaluación docente, la dimensión «Interacción Pedagógica» se evalúa a través de la clase filmada en el módulo 2 del portafolio, donde se analiza la Calidad de las Explicaciones que muestra cómo el docente enseña a sus estudiantes nuevos conocimientos y la vinculación de estos con aprendizajes previos, el tipo de preguntas que formula así como los ejemplos que entrega para clarificar las dudas de sus estudiantes, y por otro lado la Calidad de las Interacciones que evalúa la calidad de las preguntas del docente para fomentar el aprendizaje y cómo éste aprovecha las intervenciones de sus estudiantes para profundizar los conocimientos en la clase (CPEIP, 2013).

En el proceso de evaluación docente, un docente "Competente" en esta dimensión, debe ser capaz de organizar situaciones interesantes y 
productivas que favorezcan la indagación, interacción y socialización de los aprendizajes entre los estudiantes, en situaciones planteadas desde las competencias de los estudiantes, es decir, de los saberes e intereses de estos, fomentando la posibilidad de desarrollo del pensamiento y de habilidades de elaboración cognitiva más complejas (Manzi, J. et al., 20I I).

El aula es el espacio que debe garantizar oportunidades de desarrollo para todos los estudiantes debido a que estos dependen críticamente de la capacidad del profesor para ser un mediador efectivo en la construcción de nuevos conocimientos (De Corte, 2010). En este sentido, se considerará que las interacciones pedagógicas serán de calidad cuando el profesor, en un proceso de retroalimentación continua, recoge la información desprendida del resultado de su trabajo y retroalimenta las intervenciones de sus estudiantes, reflejando el avance hacia el objetivo de aprendizaje propuesto para la clase (Collins, 2006).

La interacción dentro de la sala de clases ha sido uno de los ejes de trabajo del Ministerio de Educación desde el año 2003, en donde comprender este proceso es un factor clave para el desarrollo de los aprendizajes (Murillo \& Román, 2009), ya que la interacción entre profesor y estudiante es un complejo proceso de razonamiento interpersonal en el aula que tiene como única finalidad la constitución de nuevos aprendizajes (Velasco, 2007).

\section{La teoria de la modificabilidad cognitiva y experiencia de aprendizaje mediado}

La interacción docente-estudiante está ligada al desarrollo cognitivo a través del diálogo, compartiendo saberes y formas de ver el mundo. Los enfoques socio-cognitivos del aprendizaje y de la enseñanza proponen que el desarrollo cognitivo es un proceso culturalmente organizado, en el cual los procesos psicológicos superiores emergen a partir de la apropiación y dominio de los instrumentos y recursos que la cultura posee, a través de la interacción humana (Baquero, 2004). Estos enfoques aportan sobre el vínculo entre el conocimiento y el contexto en que éste se adquiere: desde los procesos mediacionales, 
que relevan el rol del educador y, por otra parte, la mediación semiótica, que aporta a la construcción intersubjetiva de significados (Pasmanik y Cerón, 2005).

Feuerstein (2006) profundiza en las formas en que se dan los procesos de mediación, enfatizando en el rol que ejecuta el mediador humano en la interacción. Señala que los procesos de aprendizaje son potencialmente transformadores cuando existen Experiencias de Aprendizaje Mediado. La EAM se refiere a la forma en que un estímulo es transformado por un docente mediador, de acuerdo a una intención clara y metas que apuntan a alcanzar un funcionamiento cognitivo superior. Es una interacción que apunta a la transformación del desempeño de los sujetos en una tarea y que parte de la comprensión del funcionamiento cognitivo como la manifestación de un estado del organismo en un momento de la vida y no como una condición estable (Feuerstein \& Kozulin, I995). Así, la Experiencia de Aprendizaje Mediado es una modalidad pedagógica que potencia el desarrollo cognitivo a través de las interacciones entre el docente y el estudiante. El docente-mediador debe apuntar al desarrollo de aprendizajes significativos por lo que el sentido de quien enseña no sólo debe estar puesto en impartir información, sino que en entregar definiciones, instrucciones claras e intención (Feuerstein R., 1996).

El aula es un lugar propicio para desarrollar experiencias de aprendizaje mediado, ya que está lleno de estímulos constituidos por objetos e interacciones que deben ser transformadas por la intención y anticipación del docente, que no sólo debe presentar información acerca de lo que desea trabajar en su clase, también debe crear contextos en que esta información sea percibida, registrada y elaborada en los marcos de tiempos y espacios que le permitan a los estudiantes crear un significado particular y propio que vaya más allá de la misma experiencia concreta (Feuerstein \& Feuerstein, 199I).

Desde la perspectiva educacional el aporte del rol mediador otorga al docente la posibilidad de actuar intencionadamente para develar el potencial cognitivo y social de sus estudiantes, como también 
permite favorecer espacios de encuentro dialógico y aprendizaje colaborativo entre pares. En este rol hay plena conciencia de las posibilidades que tiene como mediador en los procesos de enseñanza y aprendizaje, como se produce el pensamiento y aprendizaje de sus estudiantes y los mecanismos que los intervienen, mostrando experticia en técnicas y estrategias de aprendizajes para saber cómo intervenir de forma correcta en la solución de las debilidades que encuentra en sus estudiantes durante la interacción (Tébar, 20I2). El docente, entonces, modifica un conjunto de estímulos afectando su intensidad, contexto, frecuencia y orden, buscando despertar en sus estudiantes una actitud vigilante, consciente y sensible que contribuya a desarrollar una genuina disposición al aprendizaje autogestionado.

La función pedagógica como un acto mediacional y de modificabilidad cognitiva va más allá del simple traspaso de contenidos (Tebar, 2009), entendidos como formas de saber, y los métodos (formas de hacer) (Román \& Díez, 2008). El docente mediador es, entonces, un constructor de puentes entre el medio y el niño, posibilitando la incorporación de una amplia gama de estrategias cognitivas y procesos, que derivarán en comportamientos considerados como pre-requisitos para un buen funcionamiento cognitivo y afectivo (Tebar, 2012). Esto exige tanto en el docente como en el estudiante una fuerte actividad intelectual, la que pese a ser propia de cada sujeto no se hace de forma individual, sino que es el fruto de la interacción entre docente y estudiantes.

No cualquier interacción entre el mediador y el sujeto puede ser considerada una experiencia de aprendizaje mediado, por ello para que el actuar del docente sea calificado como una acción mediadora se deben cumplir con algunos criterios relativos a su proceder en el aula. De este modo, el profesor Feuerstein (1986; 1996) plantea como necesaria la presencia de tres características fundamentales o Criterios de Mediación Universales:

- Criterio de Intencionalidad y Reciprocidad: En la interacción el docente comparte el propósito de la interacción de forma explícita y consciente, buscando una respuesta recíproca en los estudiantes. 
- Criterio del Significado: Consiste en dar sentido a los contenidos y a las alternativas pedagógicas buscando el porqué del razonamiento y la forma lógica de expresar el pensamiento.

- Criterio de Trascendencia: Significa ir más allá del aquí y ahora de la situación o necesidad inmediata que motivó la interacción. Cada situación producida en una interacción potencialmente puede ser útil en otras situaciones de vida.

Aparte de estos Criterios de Mediación Universales, Feuerstein (I986; I996) agrega otros Criterios de Mediación Diversificadores que responden a características culturales de la interacción y que son los responsables de la diversificación de estilos cognitivos del aprendiz; entre ellos podemos encontrar mediación del sentimiento de competencia, mediación de la regulación y control del propio comportamiento, mediación del acto de compartir, mediación de la individualización y diferenciación psicológica, mediación de búsqueda, planificación y logros de los objetivos de la conducta; mediación del desafio; mediación del ser bumano como entidad cambiante; mediación de la alternativa optimista; mediación del sentido de pertenencia.

En este contexto, este artículo busca profundizar en las interacciones pedagógicas de profesores categorizados como Competentes y Básicos en el proceso de evaluación docente, desde el enfoque de la EAM.

\section{Método}

La presente investigación es de carácter comparativo y de alcance descriptivo, con un enfoque metodológico mixto (Tashakkori \& Teddlie, 2003) para el análisis de las Interacciones Pedagógicas desde los Criterios de Mediación de la Teoría de la Modificabilidad Estructural Cognitiva (Feuerstein \& Feuerstein, I99I).

La muestra, de tipo intencionada, fue de 6 docentes del sistema de educación municipal de la Comuna de Los Vilos, quienes fueron evaluados a través del Sistema de Evaluación Docente. Los profesores corresponden al nivel de Enseñanza General Básica, nivel que constituye el $92 \%$ de la oferta educativa del Departamento de Educación Municipal de esta comuna. De los 6 docentes, y 
para fines comparativos, 3 de ellos obtuvieron la calificación de competentes y 3 fueron evaluados como Básicos, por otra parte se aportó variabilidad al incorporar docentes por cada tipo de escuela de enseñanza general básica presente en la comuna (urbana, rural, y multigrado).

Las Interacciones Pedagógicas fueron registradas en 3 clases videadas, de 45 minutos para cada docente, en los sectores de lenguaje y matemáticas. Las filmaciones fueron analizadas a partir de la pauta de observación de criterios de mediación en aula desarrollada por Salinas y Dávila (2013), pauta validada con un índice de Kappa de 0,934 (Salinas, 20I3). La pauta define para cada uno de los I2 criterios de mediación, entre 2 a 3 indicadores que deben ser evaluados de acuerdo a tres niveles de calidad de las mediaciones.

Figura I: Criterios de Mediación e Indicadores correspondientes a cada Criterio observado.

\begin{tabular}{|l|l|}
\hline $\begin{array}{l}\text { CRITERIOS DE MEDIACIÓN } \\
\text { UNIVERSALES }\end{array}$ & INDICADORES \\
\hline $\begin{array}{l}\text { I. Mediación de la } \\
\text { Intencionalidad y Reciprocidad }\end{array}$ & I. Explicitación de objetivos. \\
\cline { 2 - 2 } & 2. Adaptación de los estímulos. \\
\hline $\begin{array}{l}\text { 2. Mediación de la } \\
\text { Trascendencia. }\end{array}$ & I. Establecimiento de relaciones. \\
\cline { 2 - 2 } 3. Mediación del Significado. & $\begin{array}{l}\text { 2. Trasferencia de contenidos. } \\
\text { del contenido por parte del docente. }\end{array}$ \\
\cline { 2 - 2 } & $\begin{array}{l}\text { 2. Estudiantes explicitan significado } \\
\text { afectivo del contenido. }\end{array}$ \\
\cline { 2 - 2 } & $\begin{array}{l}\text { 3. Evocación y establecimiento de } \\
\text { relaciones con conocimientos previos. }\end{array}$ \\
\hline
\end{tabular}

(Salinas \& Dávila, 2013)

Estos indicadores se clasifican en nivel alto, nivel medio y nivel bajo, en relación a la aparición de estos durante sus Interacciones Pedagógicas. Los niveles se detallan a continuación: 
Figura 2: Niveles de Calidad de la Mediación Según Promedio

\begin{tabular}{|c|c|}
\hline $\begin{array}{l}\text { Nivel de } \\
\text { Mediación Alto } \\
\text { Rango } \\
2.34 \text { a } 3\end{array}$ & $\begin{array}{l}\text { Promedian entre } 2.34 \text { a } 3 \text { en la mediación de los indicadores } \\
\text { correspondientes a los Criterios de Mediación evaluados. } \\
\text { En general se observan Interacciones Docente-Estudiante en } \\
\text { las que luego de una mediación del indicador por parte del } \\
\text { docente, este logra que sus intervenciones generen cambios } \\
\text { observables en los estudiantes. }\end{array}$ \\
\hline $\begin{array}{l}\text { Nivel de } \\
\text { Mediación } \\
\text { Medio } \\
\text { Rango } \\
\text { I.67 a } 2.33\end{array}$ & $\begin{array}{l}\text { Promedian entre } 1.67 \text { y } 2.33 \text { en la mediación de los } \\
\text { indicadores que corresponden a los determinados } \\
\text { Criterios de Mediación evaluados. En general se observan } \\
\text { interacciones Docente-Estudiante en las que luego de } \\
\text { una mediación del Indicador por parte del docente no se } \\
\text { observan cambios observables en los estudiantes. }\end{array}$ \\
\hline $\begin{array}{l}\text { Nivel de } \\
\text { Mediación Bajo } \\
\text { Rango } \\
\text { I a I.66 }\end{array}$ & $\begin{array}{l}\text { Promedian entre I y I.5 en la mediación de los indicadores } \\
\text { que corresponden a los determinados Criterios de } \\
\text { Mediación evaluados. En general hay ausencia de una } \\
\text { mediación del indicador por parte del docente. }\end{array}$ \\
\hline
\end{tabular}

Dado el tamaño de la muestra, el análisis de los datos se realizó a partir de estadísticos descriptivos (medias) por cada grupo de docentes, sin llegar a establecer significación estadística en las diferencias observadas. Por otra parte, se realizó una descripción de carácter cualitativo respecto del despliegue de criterios de mediación más característicos - universales y diferenciadores- según los niveles de calidad arrojados por la pauta y a fines de caracterizar las interacciones.

\section{Resultados}

Los resultados se presentan de forma comparativa para las dos categorías de docentes -competentes y básicos-, primero según su desempeño en los criterios de mediación universales y luego para los criterios de mediación diferenciadores.

El siguiente gráfico da cuenta de las diferencias en las puntuaciones de los indicadores de los criterios de mediación universales observados en ambos grupos de docentes... 
Figura 3: Comparación de la Calidad de la Mediación entre los Profesores categorizados Competentes y Básicos según los Criterios de Mediación y sus indicadores.

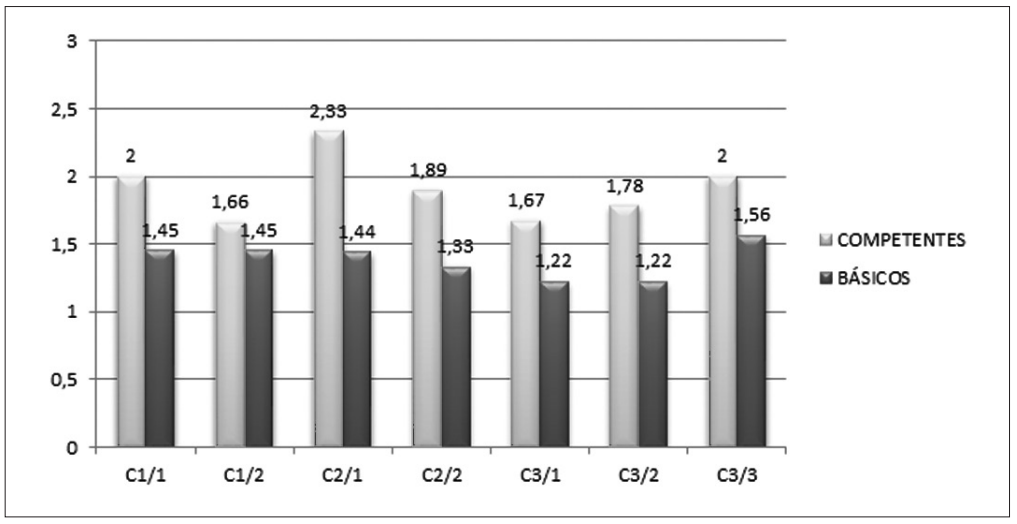

\subsection{Criterios de mediación universales}

\begin{tabular}{|l|l|l|}
\hline & COMPETENTES & BÁSICOS \\
\hline $\begin{array}{l}\text { I. Mediación de la } \\
\text { Intencionalidad y Reciprocidad }\end{array}$ & Medio & Bajo \\
\hline $\begin{array}{l}\text { 2. Mediación de la } \\
\text { Trascendencia. }\end{array}$ & Medio & Bajo \\
\hline 3. Mediación del Significado. & Medio & Bajo \\
\hline
\end{tabular}

\section{Resultados docentes competentes}

La mediación de la Intencionalidad y la Reciprocidad en los docentes competentes es de nivel medio ( $=$ I.8I) con una desviación estándar de 0.45. De los tres profesores, dos de ellos buscan explícitamente que los estudiantes realicen de forma adecuada procedimientos $y$ mecanismos de resolución de problemas matemáticos. Esto se puede observar de mejor forma en la interacción que propone el docente 2: "Quiero que bagan un rectángulo porque luego vamos a trabajar con sus medidas y determinaremos el área de esta figura", o por ejemplo lo que ocurre con la segunda clase del docente 3; "Esto, lo que trabajaremos se llama intervalo y lo calcularemos de esta forma y nos sirve para determinar algunas frecuencias entre los datos". El otro docente que se ubica entre un nivel de calidad alta en este criterio $(=2.33)$, desde el comienzo deja claro en sus 
alumnos lo que desea conseguir con su actividad: "Vamos a analizar el cuento escuchado, por lo que deberán estar muy atentos a lo que escuchan y les aconsejo que presten atención a las características del protagonista" (Docente I, Clase I). En esta clase el docente hace explícito el objetivo de la experiencia y la intencionalidad logra ser comprendida por los estudiantes (comprender un texto de mediana complejidad a través de una estrategia de anticipación de la lectura).

En el otro indicador de este criterio el nivel de calidad de la mediación se invierte. Los dos que en un comienzo presentan un bajo nivel de mediación evidencian un nivel alto de calidad en la mediación de adaptación de los estímulos. A modo de ejemplo en la primera clase del docente 2 ocurre lo siguiente: "Ya sé por qué se ban equivocado, parece que el segundero es el que los ba confundido pero por abora lo vamos a dejar de lado porque lo que quiero es que comprendamos primero cómo se van indicando las boras y los minutos en un reloj análogo" (Docente 2, Clase I). Esta interacción muestra un nivel de calidad alto de la mediación sobre los elementos que componen la tarea que se está desarrollando para que estos puedan ser asimilados y comprendidos por todos los estudiantes. Son capaces de descomponer los elementos e ir paso a paso una vez más. Al Docente I se le dificulta acercar al alumno la información inferencial que contiene el texto que está trabajando. Evidencia dificultades para dilucidar el origen de las dificultades que presentan los estudiantes para acceder a la información, resolviendo la situación problemática traspasando de forma directa la información a la que no logran acceder, como por ejemplo "Vieron, ¿Ustedes cuántas veces ban sido igual que el gato del cuento con sus mamás, porfiados, desobedientes?” (Docente I, Clase 2).

En el otro Criterio de Mediación Universal restante, Criterio de Mediación del Significado, la calidad de la mediación es de nivel medio ( = I.8I) con una desviación estándar de 0.36 producto de que existe uno de los docentes con una calidad de la mediación baja en este Criterio.

La calidad de la mediación de los Indicadores para este Criterio durante las Interacciones de estos profesores en sus clases es muy distinta, sobre todo en las de Docente I, en los tres Indicadores pero 
sólo en la primera clase. Por ejemplo en su primera clase el Docente I trabaja la comprensión de lectura mediante un texto que posee un fuerte contenido formativo en lo valórico. El texto se llama el "Gato Desordenado" y la interacción comienza de la siguiente manera: " $A$ ver, ¿de qué creen ustedes que se trata el texto Gato Desordenado? Luego de esta pregunta el docente genera el espacio para que los alumnos respondan como forma de anticipación a la lectura a partir de sus experiencias previas tales como: "Se trata de un gato desordenado que no le bace caso a nadie". Esta opinión por parte de los alumnos, que recordemos está al inicio de la clase, le permite al docente realizar una vinculación del contenido con elementos afectivos que identifican al alumno con el protagonista de la historia que han leído. En la misma clase el docente I le dice a sus alumnos luego de realizar la lectura: "Vieron, se parece en algo a lo que ustedes creían de lo que se trataba el cuento... (a lo que los alumnos responden que si) ...Y a ver, a cuántos de ustedes sus mamás les dan la oportunidad de aprender una y otra vez aunque se equivoquen tal cual como lo bizo la mamá del gatito".

El promedio de la calidad de la mediación de los otros dos docentes es inferior respecto a Docente I. El foco en la mediación de estos docentes está puesto en establecer la relación casi mecánica de lo que trabajarán en esta nueva clase con el contenido visto en la clase anterior. Un ejemplo de esto en el docente 2: "Se acuerdan cómo calculábamos el área de las figuras geométricas, bueno, para calcular el área en un cuerpo geométrico es algo similar". Por otro lado el docente 3, para generar un vínculo del contenido con elementos afectivos, raya en lo peyorativo, puesto que del contenido que se está trabajando, que son los índices de obesidad de la población escolar de la comuna de Los Vilos, el docente realiza el siguiente comentario: "Como pueden ver no es una característica muy buena el ser la escuela con el mayor índice de obesidad de la comuna".

\section{Resultados de los docentes básicos:}

En el Criterio de la Mediación de la Intencionalidad y Reciprocidad (Criterio de Mediación Universal), requisito de toda Experiencia de Aprendizaje Mediado, su calidad de la mediación es baja ( = I.45) con una desviación estándar de 0.39. Cabe aclarar que influye en 
esto que en la primera clase uno de los tres docentes ofrece una calidad de la mediación mayor. A modo de ejemplo, el Docente 5 en su primera clase ofrece una mediación de alta calidad donde explicita el objetivo de su clase: "Vamos a escuchar este cuento con mucha atención porque al final aprenderemos la palabra nueva”... así como también realiza una adaptación del contenido que está trabajando para que todos sus alumnos lo asimilen; "Aquí en esta parte del cuento debo cambiar la voz porque bay un signo de interrogación y es en donde el sapito le pregunta al abuelo" (ID5-CI). Los otros dos docentes (D4 y D6) ofrecen un nivel de mediación de muy baja calidad. Por un lado el Docente 4 lee el objetivo de la clase escrito en la pizarra y luego de eso dice al curso: "Vamos a escuchar para comprender la lectura" (Docente 4, Clase I). Al decir "Comprensión” en la instrucción da a entender de manera implícita que por ese solo hecho ya los alumnos tendrán que comprender lo que ella les leerá. En la interacción del Docente 6 sus intervenciones no califican para poder hablar de una mediación de este Criterio, ya que realiza claramente una instrucción directa, "Van a pegar las oraciones y luego van a leer para que puedan responder estas preguntas" (ID6-CI). Ya en la última clase los tres docentes de esta categoría finalizan con este tipo de interacciones caracterizadas por la instrucción directa y cerrada hacia una solución.

En cuanto a la calidad de la Mediación de la Trascendencia (Criterio de Mediación Universal) que estos docentes ofrecen a sus alumnos, es de bajo nivel (= I.39) con una desviación estándar de 0.IO. El Docente 4 está trabajando un texto referido al "Cochayuyo" que busca cumplir dos objetivos de manera simultánea: la comprensión de lectura a partir de un texto escuchado, ya que por ser primero básico gran parte de los alumnos no leen de manera independiente, y por otro lado la producción escrita de palabras con " $\mathrm{CH}^{\prime}$. Luego de haber iniciado la clase, y sin haber establecido relaciones previas a la lectura con experiencias de la vida cotidiana, comienza por entregar una serie de informaciones donde ni siquiera espera a que algún alumno conteste... ¿Algunos de ustedes ha comido cochayuyo? Sirve para muchas cosas, por ejemplo cuando le están saliendo los dientes a sus hermanitos. ¿Han visto a sus hermanitos cómo sufren cuando les están saliendo los dientes?... Y bueno, también estamos leyendo 
este cuento porque la palabra cochayuyo tiene la letra que vamos a estudiar hoy, la letra CH” (Docente I, Clase I).

Por otra parte, el docente 5 trata de establecer una relación al comienzo de su segunda clase con lo visto en la anterior pero al preguntar si se acuerdan de qué se trataba el texto y la palabra que habían "aprendido" la clase anterior, solo un alumno la recuerda. Al notar esto comienza a rememorar parte de la clase anterior y dice: "Vimos la palabra VALIENTE y yo les daré un ejemplo de la palabra: Pedrito fue Valiente el otro día porque se vacunó y no lloró" (ID5-C2). En cuanto a las clases del Docente 6, se observan atisbos de mediación del Criterio de la Trascendencia pero esto no queda en más que en una relación muy general, basada en algún aspecto de la tarea a trabajar pero sin relación con el objetivo de aprendizaje, por ejemplo: "Abora les voy a pasar una boja que yo sé que les va a gustar mucho porque se trata de fútbol" (ID6-E2).

Finalmente, la calidad de la Mediación del Significado no es mejor que los criterios de mediación anteriores ya que igual es baja (= I.33), con una desviación estándar de 0.40. En las clases del Docente 5 no se refleja alguna interacción que pueda dar cuenta de la mediación de este Criterio así como tampoco en las clases del Docente 4. En el caso de las clases del Docente 6 se muestra algún tipo de significación enfocada a ellos como personas... "Los números en la espalda de los jugadores de fútbol sirven para identificar al jugador y la posición que le corresponde dentro de la cancha, como cuando Ustedes juegan a la pelota”... pero vuelve a quedar en una significación tal cual como la transferencia que hace. Se centra en la actividad pero no en el objetivo de aprendizaje, lo cual termina desvirtuando la clase hacia otro rumbo.

\section{Análisis comparativo}

Para los 3 criterios de mediación universales, a saber, Intencionalidad y Reciprocidad, Trascendencia y Significado (CI, C2 y C3) los docentes evaluados como competentes muestran un nivel medio de calidad, mientras que los docentes evaluados como básicos, se sitúan en un nivel bajo, según los rangos señalados en la pauta. En términos generales, los docentes competentes hacen explícito aquello que 
desean conseguir en sus estudiantes pero sin lograr una comprensión profunda acerca de su intención pedagógica.

Los docentes competentes investigados fomentan la comparación entre objetos pero los estudiantes no logran establecer las relaciones aun cuando guían de buena manera la transferencia de los contenidos. Sus estudiantes no visualizan una posible aplicación del mismo a otros contextos siendo que el objetivo es explícito. Por otra parte, los docentes básicos no explicitan qué es lo que quieren conseguir, se limitan sólo a leer el objetivo escrito en la pizarra o a dictar para que este sea copiado en el cuaderno, no fomentan la comparación entre diferentes objetos o características de los estímulos, así como no guían transferencias ni siquiera a nivel de contenidos que se están trabajando o se han visto anteriormente en otras clases.

En términos cualitativos, la calidad de la mediación de la Intencionalidad que desarrollan los profesores competentes no es constante en las tres clases. Se observa que en las dos primeras se ofrece una mediación de mayor calidad, la cual decae en la tercera clase equiparándose con la de los profesores básicos. Los docentes competentes, en general hacen explícito qué es lo que desean conseguir en sus estudiantes, sin embargo, éstos no logran decodificar su intención pedagógica, no logrando involucrarse en el proceso de enseñanza propuesto.

En la tercera clase los docentes, tanto competentes como básicos evidencian una ausencia casi total de mediación de este criterio ya que no explicitan qué es lo que quieren conseguir, se limitan sólo a leer el objetivo escrito en la pizarra o a dictar para que este sea copiado en el cuaderno. Tampoco adaptan los estímulos para favorecer la apropiación conceptual de los estudiantes.

En cuanto a la Trascendencia los docentes competentes durante sus clases fomentan la comparación entre elementos relacionados con la actividad de aprendizaje pero los estudiantes no logran establecer las relaciones, no visualizan una posible aplicación del mismo a otros contextos. Los profesores básicos en cambio no fomentan la comparación entre diferentes objetos o características de los estímulos, como tampoco guían transferencias ni siquiera a nivel de 
contenidos que se están trabajando o de contenidos abordados en clases anteriores.

En la mediación del Significado, los profesores competentes realizan alusiones al significado personal que para cada quien tiene la tarea y preguntan a sus estudiantes acerca de la importancia del tema, sin embargo, para los estudiantes no pasa de ser un contenido aislado que se ubica sólo en el contexto de una actividad formal, ajena a la vida cotidiana de ellos, sin una carga emotiva y sin espacio para expresar sus conocimientos previos.

La baja calidad de la Mediación del Significado de los docentes básicos se manifiesta en que estos no consideran explícitamente los conocimientos previos de los estudiantes, no otorgando un significado personal al contenido de aprendizaje ni tampoco manifestando vínculos de la materia tratada con experiencias de la vida cotidiana. Por otra parte, los docentes competentes favorecen el desarrollo de una imagen positiva en sus estudiantes, pero pese a ello no logran desbloquear el comportamiento de ensayo y error. Aquellos docentes categorizados como básicos, tampoco se preocupan de desarrollar una imagen positiva de sus estudiantes, ni propician el análisis de los procesos cognitivos realizados por sus estudiantes.

\section{Discusión y conclusiones}

A partir de los resultados expuestos se puede apreciar que las interacciones pedagógicas ofrecidas por los docentes categorizados como competentes son de mayor calidad en comparación con los profesores categorizados como básicos en los Criterios de Mediación Universales. Ahora bien, es conveniente aclarar que ninguna de las clases observadas puede ser valorada, desde el enfoque de la EAM, como una experiencia de aprendizaje mediado. Por ello, independiente de la categoría de evaluación que obtienen los docentes, ésta no predetermina un tipo de interacción de un valor mediacional alto. Visto esto, es necesario focalizar en las similitudes y diferencias de los docentes estudiados; por ejemplo, se observa que entre los profesores categorizados como básicos existe cierta similitud en las interacciones que proponen, realizando una mediación de mejor calidad en la primera clase, con un patrón 
de interacción que podríamos relacionar con la "clase filmada" de la evaluación docente, pero que se disipa completamente hacia la tercera clase en términos de calidad. Así mientras la primera clase puede ser caracterizada como el desarrollo de un guion altamente estructurado, la tercera clase se configura quizás más desorganizada pero mucho más natural, posibilitando el espacio para actividades emergentes. Dichos docentes también presentan una dificultad evidente en el establecimiento de relaciones propias del contenido curricular y aspectos de la vida cotidiana de sus estudiantes: no propician interacciones desde la trascendencia, se centran en la actividad pedagógica pero no consideran el objetivo de aprendizaje, lo cual termina desenfocando la clase, lo que se nota especialmente durante la tercera clase.

El hecho de que la metodología de esta investigación haya propuesto tres clases filmadas y no una, permite identificar modalidades «típicas» de interacción pedagógica, aquellas que se reiteran dando cuenta de prácticas inherentes a su labor docente, por una parte, y de inconsistencias en la calidad de la mediación entre las tres clases, por otra. Aquel docente que muestra mayor desempeño en la primera clase en comparación a la tercera, refleja que en la primera clase ha realizado algo que no es habitual en su práctica, lo que coincidentemente se asemeja a la estructura de la evaluación docente. Un resultado llamativo, es que de los tres docentes categorizados como básicos, aquel que se desempeña en aula multigrado muestra un desempeño estable en las interacciones de sus tres clases y es quien, por momentos, durante las mismas se acerca a una Experiencia de Aprendizaje Mediado.

En términos globales, el tipo de Interacción Pedagógica que propician los seis docentes busca el aprendizaje de sus estudiantes a través de la exposición directa a los estímulos (Aprendizaje Directo), lo que desde la óptica de Feuerstein (I99I) no contribuye a generar autonomía en los procesos de aprendizaje, ya que el foco no está puesto en el desarrollo de una habilidad o interés del estudiante, sino que sólo en el contenido curricular. Este tipo de interacción fomenta un aprendizaje reducido, instrumental, y una insuficiente capacidad para resolver problemas con repercusiones serias e identificables en 
los estudiantes en todas las áreas del proceso de aprendizaje, tanto a nivel de los aprendizajes formales emanados del currículum, como de las relaciones personales en general (Feuerstein, et al., 2002). La manera de enseñar descrita en la investigación señala una brecha de acuerdo a lo propuesto por el MINEDUC (2013), relativo a la formación de estudiantes inquisitivos, equilibrados, reflexivos, audaces, comunicadores, respetuosos y empáticos, que comprenden los principios del razonamiento moral, abiertos de mentes y por sobre todas las cosas, "pensadores".

Al respecto cabe preguntarse sobre ¿cómo formar estudiantes "pensadores" si no se propicia una formación docente inicial y continua consistente con estos principios? ¿Cómo estimular "estudiantes inquisitivos" si no se le otorga la suficiente relevancia al desarrollo de interacciones pedagógicas desafiantes y transformadoras, por sobre interacciones mecanizadas y reproductivas?

En todos los casos investigados se evidencia baja presencia de interacciones que potencien aquellas funciones cognitivas requeridas para la recogida, elaboración y comunicación de la información, necesarias para el surgimiento de operaciones mentales en los estudiantes (Feuerstein, 2006; Villalta, et al., 2013). Esto hace necesario reflexionar, no solo sobre la función docente, sino que sobre el fin práctico de nuestra educación y en particular sobre sus sistemas evaluativos y de desarrollo profesional en cuanto a su idoneidad para desarrollar el pensamiento, a través de las interacciones.

Aun cuando las nuevas bases curriculares dan por supuesto al currículum como herramienta para el desarrollo del pensamiento (Ministerio de Educación, 2013), persiste una brecha en esta materia. La tensión entre currículum prescrito y práctica pedagógica, releva la importancia de la evaluación y análisis de la práctica pedagógica, así como la de interrogar la propia acción docente. Cuando los docentes son conocedores de los beneficios de la reflexión sistemática y la investigación, y participan de experiencias formativas que propicien un desarrollo profesional sustantivo, se convierten en agentes activos del cambio en su propia aula y en su entorno próximo (Pellerin \& Faukner, 2015). Sin embargo, y mientras tanto, al momento 
de hacer sus clases los profesores parecen priorizar sobre todo la cobertura curricular, declarando múltiples razones para ello, siendo la más relevante el desempeño demostrable: muchas veces la clave es el puntaje a obtener en el SIMCE. Implícitamente, parece existir la idea de que el "pasar la materia" establecida en los planes y programas producirá, por sí mismo, un mejor rendimiento en dicha prueba, reflejando una dualidad entre desarrollo del pensamiento y contenido curricular.

El hecho de dominar la disciplina que el docente enseña, pasa a ser un aspecto relevante en este ámbito, mas no suficiente. Manejar la disciplina propia del sector de aprendizaje resulta relevante a la hora de diseñar y de interactuar desde la anticipación y dominio pedagógico, sin embargo, la expertise de construir conocimiento pedagógico con los estudiantes se transforma en un saber de difícil gestión. En términos globales, las clases observadas son tradicionales, no fomentan la participación ni ofrecen instancias de complemento a los conceptos abordados y de profundización de los aprendizajes (Manzi, et al., 20I I).

Las interacciones estudiadas carecen de un foco claro, lo que como efecto secundario expone a los estudiantes a un repertorio limitado de intereses, dificultando la generalización de sus aprendizajes a contextos más remotos. La posibilidad de diversificar estilos de interacción permite, por tanto, activar funciones cognitivas claves para el desarrollo humano y la adaptación a una variedad de cambios técnicos, sociales y culturales en un mundo que está en constante cambio (Lebeer, 2005).

Ahora bien, podemos preguntarnos en qué medida la demanda del sistema sobre los resultados de aprendizaje y la tendencia "Univocista" y "Mecanicista" de la evaluación docente (Monzón, 2016), predefinen modalidades estandarizadas de hacer clases que no permiten ahondar en la indagación colaborativa y la interacción ligada a la construcción del conocimiento pedagógico. Los resultados aquí expuestos, aunque circunscritos a los casos estudiados, invitan a considerar la necesidad de observar la calidad de los procesos educativos, más allá de las lógicas de accountability y efectividad 
escolar (Romero-Farfán, 2007; Assael, 2014), propiciando procesos de reflexividad y autobservación en los docentes (Figueroa y Gómez, 2015). Aspectos relacionados con lo anterior, dicen relación con estudiar el impacto de las condiciones laborales en términos de espacios y tiempos de planificación (MINEDUC, 20I5), tanto como las instancias de desarrollo profesional, con un marcado foco en la transmisión de conocimiento más que en la indagación colaborativa y reflexiva (Ávalos, 2007; Pellerin \& Paulkner, 2015; Figueroa, 2016).

La interacción pedagógica debiera posicionarse con fuerza en los procesos formativos y evaluativos de nuestros docentes, trascendiendo el aprendizaje directo y enfoques tradicionales, reproductivistas e instruccionales. Desarrollar el rol mediador docente, desde esta interacción, implica un interjuego transformador entre el currículum prescrito, la intencionalidad docente y la participación de los estudiantes (Figueroa, 2016), por lo que alinear sentidos en relación a las reformas implica necesariamente propiciar una reflexividad consciente, afianzando un desarrollo profesional genuino y transformador desde la base. Esto implica, indiscutiblemente, alinear la política educativa (ley de inclusión, diversificación curricular, bases curriculares, etc.) y las intencionalidades más profundas del proceso de evaluación docente, de modo tal de propiciar un cambio integral, analógico y situado, desde el significado y trascendencia de la función educativa.

\section{Referencias bibliográficas}

Assael, C. (2014). La Educación de personas con discapacidad en las escuelas regulares: discurso de la valoración de la diferencia, bajo el enfoque de una cultura hegemónica. En E. López, Inclusión de la Vida y la Escuela: Pedagogía con Sentido Humano (págs. 27-42). La Serena: Universidad de La Serena.

Assaél, J. (2008). VII Seminario Redestrado - Nuevas Regulaciones en América Latina. Políticas Educativas y Trabajo Docente en Chile. Buenos Aires, Argentina.

Ávalos, B. (2007). El desarrollo profesional continuo de los docentes: lo que nos dice la experiencia internacional y de la región latinoamericana. Revista Pensamiento Educativo, 4l; N², 77-99. Retrieved from http:// www.pensamientoeducativo.uc.cl/files/journals/2/articles/4I7/ public/4I7-934-I-PB.pdf 
Bonal, X. (1998). La política educativa: dimensiones de un proceso de transformación (1976-I996). Políticas públicas en España. BarcelonaEspaña: Contenidos, redes de actores y niveles de gobierno.

Burger, M. (2004). La gestion des activités: pratiques sociales, rôles interactionnels et actes de discours. Cahiers de Linguistique Francaise. Obtenido de http://clf.unige.ch/files/26/I2-Burger.pdf

Colegio de Profesores de Chile A.G. (2000). La complejidad de las políticas de evaluación del desempeño docente. Docencia, I0-53.

Collins, A. (2006). Cognitive apprenticeship. En R. Sawyer, The Cambridge bandbook of the learning sciences (págs. 47-60). New York: Cambridge University Press. C.

CPEIP (2008). Marco para la Buena Enseñanza. Santiago de Chile: Ministerio de Educación de Chile. Séptima Edición.

CPEIP (2013). Docente Mas. Obtenido de http://www.docentemas.cl/dm04_ instrumentos_port.php

Cuadrado, I. \& Fernández, I. (2008). Nuevas Competencias del Profesor en el EEES: Una experiencia de innovación docente. Revista Electrónica Teoría de la Educación. Educación y Cultura en la Sociedad de la Información.

DAEM LOS VILOS (2012). Plan Anual de Educación Municipal. Los Vilos.

Darling-Hammond, L. (2012). Desarrollo de un enfoque sistémico para evaluar la docencia y una enseñanza eficaz. Pensamiento Educativo. Revista de Investigación Educacional Latinoamericana, I-20.

De Corte, E. (2010). Historical developments in the conception of learning. In Innovative. En D. Istance, The Reader on Learning. Paris: OCDE-CERI.

Feuerstein, R. (1986). Mediated Learning Experience. Jerusalen: Hadassah Wizo Canada Research Institute.

Feuerstein, R. (I996). La experiencia de aprendizaje mediado. Langeveld conferencia conmemorativa. Utrecht: Universidad Utrecht.

Feuerstein, R. \& Feuerstein, S. (I99I). Mediated Learning experience: A theorical review. En R. Feuerstein, P. Klein, \& A. Tannebaum, Mediated Learning Experience: Theorrtical, psychosocial and learning implications. Tel Aviv \& Londres: Freund.

Feuerstein, R. \& Kozulin, A. (1995). The Bell Curve: Getting the facts straight. Educational Leadership, 52 (7), 7I-74.

Feuerstein, R., Falik, L., Rand, Y. \& Feuerstein, R. (2002). The dynamic assessment of cognitive modifiability. Jerusalem: ICELP Press. 
Figueroa, I. (2016). Rol mediador de aprendizajes en Educación Parvularia: procesos de apropiación en el contexto de una propuesta formativa en aprendizaje mediado. SUMMA Psicologica UST, 13, 33-44. http://doi. org/I0.I8774/summa-volI3.numI-287

Figueroa, I. \& Gómez, M. P. (2015). Cuestionar y problematizar la propia práctica: Investigación Acción Transformadora en los procesos de desarrollo profesional docente. Summa Psicológica, 12, 3I-42. http:// doi.org/IO.I8774/summa-volI2.num2-258

Figueroa, I., Soto, J. \& Sciolla, B. (2016). Dinámicas de recepción , apropiación y contextualización del enfoque de la Guía para la inclusión educativa en escuelas municipales de una comuna de la Región Metropolitana. Pensamiento Educativo: Revista de Investigación Educacional Latinoamericana, 53(I), I-I3. http://doi.org/I0.7764/PEL.53.I.20I6.4

Godoy, M.F. (2015). Las preguntas de docentes como estrategia para el desarrollo de habilidades cognitivas de los estudiantes en la asignatura Historia, Geografía y Ciencias Sociales. Foro Educacional UCSH, 24, 57-76.

Lebeer, J. (2005). INSIDE: Cómo aprender a aprender en un entorno inclusivo. Madrid: FERE-CECA.

Manzi, J. (2007). Seminario Evaluación Docente en Chile. Evaluación Docente: Antecedentes, resultados y proyecciones. Santiago, Chile.

Manzi, J., González, R. \& Sun, Y. (20I I). La Evaluación Docente en Chile. MaculSantiago de Chile: Centro de Medición MIDE UC.

Martinic, S. \& Vergara, C. (2007). Gestión del tiempo e interacción profesoralumno en la sala de clases de establecimientos de Jornada Escolar Completa en Chile. Revista Electrónica Iberoamericana sobre Calidad, Eficacia y Cambio en Educación, 3-20.

Ministerio de Educación de Chile (2004). Reglamento sobre evaluación docente. Recuperado el OI de 03 de 2016, de http://www. curriculumenlineamineduc.cl/605/articles-300I3_recurso_I5.pdf

Ministerio de Educación de Chile (2013). Bases Curriculares, Educación Básica. Recuperado el OI de 03 de 2016, de http:/ / portales.mineduc.cl/usuarios/ cpeip/doc/201606221649280.ReglamentoEvaluacionDocente.pdf

Ministerio de Educación de Chile (2015a). Ley de Inclusión Escolar. Recuperado el 26 de 08 de 2016, de http:/ / www.leychile.cl/Navegar?idNorma=I078172

Ministerio de Educación de Chile (2015b). Propuesta para avanzar hacia sistema educativo inclusivo en Chile: Un aporte desde la educación especial., I-57. Retrieved from http://www. 
mineduc.cl/usuarios/edu.especial/doc/20I505 I 4 I I 09250. INFORMEFINALMESATECNICAEDESPECIAL.pdf

Monzón, L. A. (2016). Hacia un paradigma analógico de la evaluación docente. Revista Iberoamericana de Educación, 53(l)(October).

Murillo, F. \& Román, M. (2009). Mejorar el desempeño de los estudiantes de América Latina: Algunas reflexiones a partir de los resultados del SERCE. Revista Iberoamericana sobre Calidad, Eficacia y Cambio en Educación, 3-9.

OCDE (2013). Teachers for the 21 st century: using evaluation to improve teaching. Paris, France: OECD Publishing.

Pasmanik, D. y Cerón, R. (2005). Las practicas pedagógicas en el aula como punto de partida para el análisis del proceso enseñanza-aprendizaje: un estudio de caso en la asignatura de química. Estudios Pedagógicos XXXI, 02, 7I-87,

Pellerin, M. \& Paukner, F. (2015). Becoming Reflective and Inquiring Teachers: Collaborative Action Research for In-service Chilean Teachers. Revista Electrónica de Investigación Educativa, 27(3), 46-60. Retrieved from http:/ / redie.uabc.mx/volI7no3/contents-pellerin-paukner.html

Prieto, M. (1990). La práctica pedagógica en el aula: un análisis crítico. Revista Educación y Pedagogía, Vol. I, 73-92.

Romero-Farfán, L. J. (2007). Concepciones de evaluación y de evaluación docente. Cuadernos de Lingüistica Hispánica, (I0), I37-I48. Retrieved from http:/ /dialnet.unirioja.es/servlet/articulo?codigo $=3324262 \&$ info $=$ re sumen\&idioma $=\mathrm{ENG}$

Salinas, G.. (20I3). Las Interacciones profesor-alumno que se relacionan con el desarrollo de babilidad de alfabetización inicial en aulas de alto y bajo rendimiento en lenguaje de prescolares pertenecientes a sectores vulnerables de Santiago. Tesis de Magíster no publicada. Santiago, Chile: Universidad Diego Portales, Facultad de Educación, Magíster en Desarrollo Cognitivo.

Salinas, G. \& Dávila, C.G. (2013). Pauta de Observación de Criterios de Mediación. Documento de Trabajo, Magíster en Desarrollo Cognitivo, Universidad Diego Portales.

Tashakkori, A. \& Teddlie, C. (2003). Handbook of mixed methods in social E bebavioral research. Thousand Oaks: Sage.

Tebar, L. (2009). El Profesor Mediador del Aprendizaje. Bogotá: Magisterio.

Tebar, L. (2012). Cómo ayudar a construir una mente bien ordenada. Conbecimiento E Diversidade, 19-28. 
Tzuriel, D. \& Haywood, H. (I99I). Interactive Assesment. New York: Library of Congress Cataloging in Publication Data.

Velasco, A. (2007). Un Sistema para el Análisis de la Interacción en el Aula. Revista Iberoamericana de Educación, I-I2.

Villalta, M. \& Martinic, S. (2009). Modelos de Estudio de la Interacción Didáctica en la Sala de Clases. Investigación Postgrado, Vol 24 n 2. PUC, 6I-76.

Villalta, M., Assael, C. \& Martinic, S. (2013). Conocimiento escolar y procesos cognitivos en la interacción didáctica en la sala de clases. Perfiles Educativos, vol. XXXV, núm. I4I, pp. 84-96, IISUE-UNAM.

Vygotski, L. (1995). Pensamiento y Lenguaje. Barcelona-España: Ediciones Paidós Ibérica, S.A. 